\title{
Liquid Expansion in Glass Sprinkler Bulbs
}

\author{
D.A. YATES, C.K. CAMPBELL, S.I. STOLIAROV, and P.B. SUNDERLAND \\ Department of Fire Protection Engineering \\ University of Maryland \\ College Park MD 20742, USA
}

\begin{abstract}
An analysis of the physics of liquid expansion in fire sprinkler bulbs is presented to characterize the variation in void size and pressure with temperature. Two types of bulbs are considered, one filled with liquid and air, and the other filled with liquid and its vapor. The analysis predicts that the void fraction decreases linearly with temperature until the void disappears or the bulb fractures. The increase of pressure at increased temperature is much steeper when there is no air in the bulb. Observations generally support the predicted void sizes, with some commercial bulbs found to contain liquid and air, and others found to contain liquid and its vapor.
\end{abstract}

KEYWORDS: activation, sprinklers, frangible bulb, suppression.

\section{NOMENCLATURE LISTING}

$\begin{array}{ll}a & \text { half width of void } \\ b & \text { half height of void } \\ \text { BP } & \text { boiling point } \\ c & \text { slope in plot of } \alpha \text { versus } T \\ f & \text { lens } f \text { number } \\ h_{f g} & \text { enthalpy of vaporization } \\ k & \text { intercept in plot of } \alpha \text { versus } T \\ \text { MW } & \text { molar mass } \\ p & \text { pressure } \\ R_{u} & \text { universal gas constant } \\ T & \text { temperature } \\ v & \text { volume }\end{array}$

$\begin{array}{ll}\text { Greek } & \\ \alpha & \text { void fraction, Eq. } 1 \\ \beta & \text { coefficient of thermal expansion } \\ K & \text { liquid compressibility } \\ \text { subscripts } & \\ 0 & \text { condition at temperature } T_{0} \\ 1 & \text { cooler than inflection } \\ 2 & \text { warmer than inflection } \\ \text { bulb } & \text { bulb } \\ \text { burst } & \text { burst (activation) condition } \\ \text { dis } & \text { disappearance } \\ \text { gas } & \text { gas } \\ \text { inflection } & \text { inflection } \\ \text { liq } & \text { liquid }\end{array}$

\section{INTRODUCTION}

Fire sprinklers are ubiquitous in fire protection and are credited with large reductions in loss of life and damage in fires. Most fire sprinklers are thermally activated. Their sensing elements are usually frangible glass bulbs, although eutectic metals are also used. Glass bulbs are also used in some pressure relief devices. Despite the widespread use of frangible glass bulbs, the physics of their activation has received no prior attention in the peer-reviewed literature.

The limited understanding of frangible glass bulb activation is evident in its published descriptions. For example, the NFPA handbook [1] states that, "The small bulb, usually of glass, contains a liquid that does not completely fill the bulb, leaving a small air bubble trapped in it. As heat expands the liquid, the bubble is compressed and finally absorbed by the liquid. Once the bubble disappears, the pressure rises substantially, and the bulb shatters, releasing the valve cap." A similar description appears in Ref. [2]. One patent describes the void as a mixture of air and gas that disappears at elevated temperature [3]. The present authors find implausible these explanations that air absorbs into liquids as described.

An improved understanding of glass bulb physics could lead to improved sprinkler performance and the use of safer liquids. For example, some bulbs contain hazardous liquids, such as halobenzenes, presenting a hazard to sprinkler installers and the environment.

The manufacture of glass sprinkler bulbs involves closely held trade secrets. Nevertheless, inspection of commercial bulbs reveals that at room temperature they all contain liquid and a gas void. In general, the larger the void fraction, the higher the burst temperature. This suggests that during manufacture the bulbs 
are filled with liquid and heated. They are then sealed either prior to or after cooling (or at an intermediate temperature). At room temperature, bulbs that were sealed prior to cooling contain the liquid and its vapor, while other bulbs contain liquid and air. When the bulbs subsequently are heated, e.g., in a fire, the liquid expands and fractures the glass.

To date, most published research on glass bulb sprinklers emphasized the plunge test and other determinations of activation temperature [4,5]. Although a plunge test yields a reliable determination of activation temperature, it provides little insight into the glass bulb physics of interest here. Others have considered the performance of sprinklers in fires, e.g., Ref. [6]. The expansion of liquids in thermometers has been considered [7], but this is a simpler process that does not involve glass rupture.

This paper begins with analytical descriptions of two glass bulb designs: one containing liquid and air and the other containing liquid and its vapor. Experiments are then presented that measure void fraction as a function of temperature for several commercial glass bulbs. Together the analysis and experiments shed light on the physics of sprinkler glass bulbs.

\section{ANALYTICAL}

An analysis is presented for two types of glass bulbs. This analysis applies to diverse liquids, such as those included in Table 1. This table includes several properties of interest here for each liquid.

Table 1. Properties of selected sprinkler bulb liquids and water. ${ }^{a}$

\begin{tabular}{cccccc}
\hline Liquid & $\boldsymbol{\beta}, \mathbf{K}^{-\mathbf{1}}$ & $\mathbf{M W}, \mathbf{g} / \mathbf{m o l}$ & $\boldsymbol{h}_{\boldsymbol{f g}}, \mathbf{J} / \mathbf{g}$ & $\boldsymbol{K}, \mathbf{M P a}$ & $\mathbf{B P},{ }^{\circ} \mathbf{C}$ \\
\hline acetone & 0.00149 & 58.1 & 98 & 800 & 56.5 \\
1,3-dicholorobenzene & 0.00094 & 147 & 178.8 & & 173 \\
glycerin & 0.00049 & 92.1 & 98.3 & 4030 & 290 \\
isopropanol & 0.00654 & 60.1 & 663.4 & 1190 & 82.5 \\
water & 0.00207 & 18 & 2258.3 & 2200 & 100 \\
\hline
\end{tabular}

${ }^{a}$ Values obtained from Refs. [8-10]. Values for $\beta, h_{f g}$, and $K$ are at $298 \mathrm{~K}$ and 1.01 bar. The BP values are at 1.01 bar.

\section{Glass Bulb Filled with Liquid and Air}

Consider a glass bulb that is filled with liquid, heated, cooled to $25^{\circ} \mathrm{C}$ and 1.01 bar such that air enters, and then sealed. The air could be replaced with any other ideal gas that does not exchange mass with the liquid. An analytical model is developed here to estimate the behavior of pressure, void fraction, and burst temperature in such a bulb. The bulb is assumed to be perfectly rigid, while surface tension and phase change are neglected. These assumptions can be relaxed, but this adds complexity.

The void fraction in the glass bulb is defined as the void volume divided by the bulb volume:

$$
\alpha=v_{\text {gas }} /\left(v_{\text {liq }}+v_{\text {gas }}\right) \text {. }
$$

The air is assumed to be an ideal gas without phase change, i.e.,

$p / p_{0}=v_{\text {gas }, 0} T /\left(v_{\text {gas }} T_{0}\right)$.

The liquid volume responds to changes in temperature and pressure such that

$v_{\text {gas }}=v_{\text {gas }, 0}-v_{\text {liq }, 0}\left[\beta\left(T-T_{0}\right)-K^{-1}\left(p-p_{0}\right)\right]$.

In most cases $K$ is large and $p-p_{0}$ small, such that the pressure term in Eq. 3 can be dropped. Assuming infinite $K$ for simplicity, and combining Eqs. $1-3$ yields the pressure inside the bulb: 


$$
\frac{p}{p_{0}}=\frac{T}{T_{0}}\left[1-\frac{1-\alpha_{0}}{\alpha_{0}} \beta\left(T-T_{0}\right)\right]^{-1} .
$$

Figure 1 depicts the behavior predicted by Eq. 4 for a glass bulb containing liquid and air. As expected, pressure in the bulb increases with increasing temperature. For a glass bulb that fractures at a certain pressure, Fig. 1 shows that the burst temperature can be selected by carefully controlling the initial void fraction, $\alpha_{0}$.

Figure 1 exhibits limiting behavior at low and at high temperatures. For low temperatures, the term in the square brackets in Eq. 4 is nearly unity, and a temperature increase causes pressure to increase primarily according to the ideal gas law. For high temperatures, the right hand side of Eq. 4 approaches infinity as the liquid volume approaches the glass volume. For such cases, there are three possible outcomes: the liquid compressibility term from Eq. 3 cannot be neglected; the bulb volume increases; or the bulb bursts.

Figure 1 indicates that pressure increases quickly with increasing temperature when $\alpha_{0}$ is low. This results in a well defined burst temperature. For large $\alpha_{0}$, the pressure rise is gradual and the burst temperature is more difficult to control.

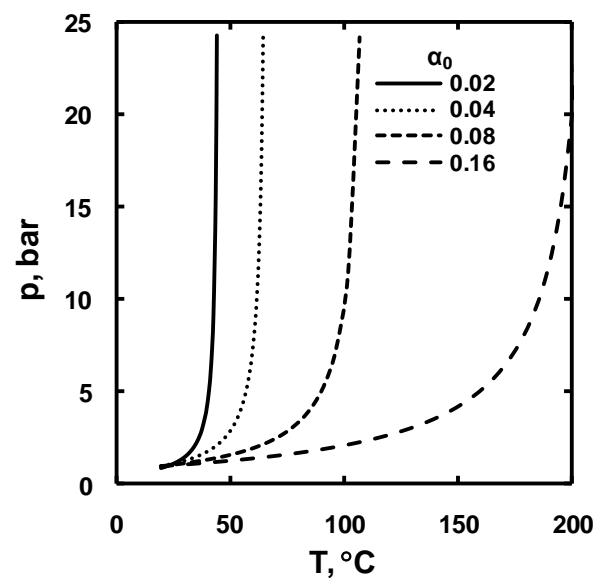

Fig. 1. Pressure as a function of temperature for a glass bulb containing liquid and air, where $T_{0}=25^{\circ} \mathrm{C}$, $p_{0}=1.01$ bar, $\beta=0.001 \mathrm{~K}^{-1}$, and $K$ is infinite.

Equations 1 and 3 can be combined to yield the void fraction,

$\alpha=\alpha_{0}-\left(1-\alpha_{0}\right)\left[\beta\left(T-T_{0}\right)-\left(p-p_{0}\right) / K\right]$.

This relationship is plotted in Fig. 2 for a glass bulb containing liquid and air (or liquid and its vapor, as discussed below). The void fraction is seen to decrease linearly with increasing temperature as a consequence of thermal expansion of the liquid. As void fraction approaches zero, pressure becomes infinite and the bulb is predicted to burst. Note that the line slopes in Fig. 2 depend on $\beta$ and, weakly, on $\alpha_{0}$.

Equation 4 allows the burst temperature to be predicted when the burst pressure is known. This temperature is expressed as

$$
\frac{T_{\text {burst }}}{T_{0}}=\frac{1+\frac{1-\alpha_{0}}{\alpha_{0}} \beta T_{0}}{\frac{p_{0}}{p_{\text {burst }}}+\frac{1-\alpha_{0}}{\alpha_{0}} \beta T_{0}}
$$


and is plotted in Fig. 3. This plot confirms the observation that an increase in initial void fraction increases the burst temperature. Note that when initial void fraction exceeds 0.2 , the burst temperature exceeds the burst temperatures of commercial glass bulb sprinklers for any reasonable burst pressure.

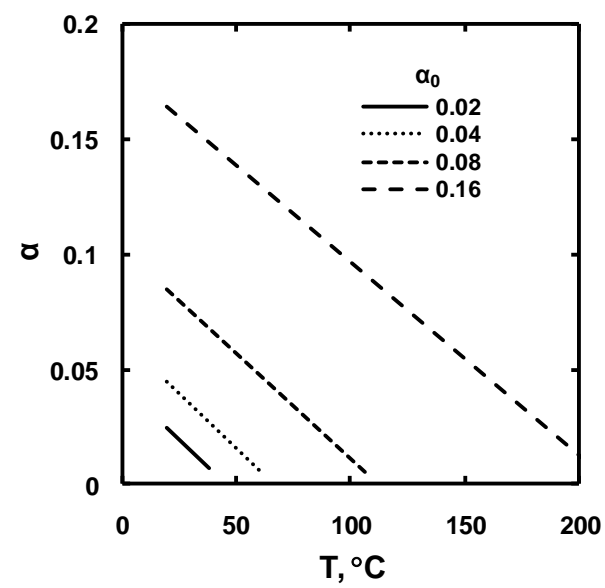

Fig. 2. Void fraction as a function of temperature for a glass bulb with an air or vapor bubble, where $T_{0}=25^{\circ} \mathrm{C}, p_{0}=1.01 \mathrm{bar}, \beta=0.001 \mathrm{~K}^{-1}$, and $K$ is infinite.

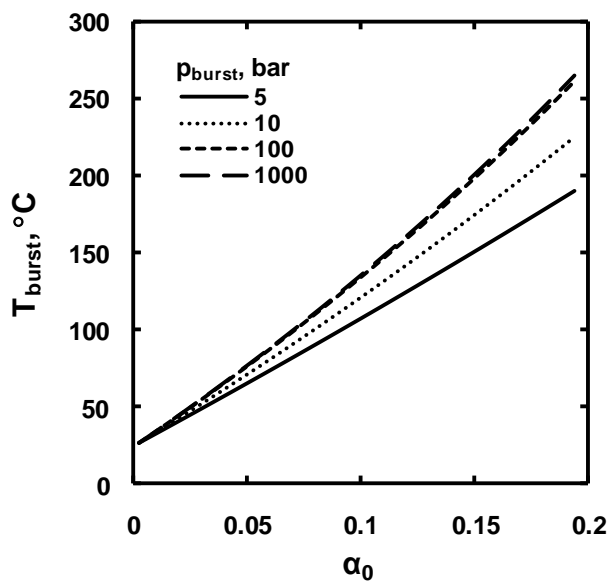

Fig. 3. Burst temperature as a function of initial void fraction for a glass bulb with an air bubble, where

$$
T_{0}=25^{\circ} \mathrm{C}, p_{0}=1.01 \mathrm{bar}, \beta=0.001 \mathrm{~K}^{-1} \text {, and } K \text { is infinite. }
$$

\section{Glass Bulb Filled with Liquid and its Vapor}

Consider next a glass bulb that is filled with liquid, heated to near its activation temperature, sealed, and then cooled to room temperature. Upon cooling the pressure drops below atmospheric and a void appears that contains the vapor phase of the liquid. No air or other gas is present inside the glass bulb. The void disappears when the pressure in the bulb reaches atmospheric pressure as a result of heating. This void collapse generates a strong compression wave, but the experiments reported below did not observe bulb rupture to coincide with void collapse.

An analytical model is developed to estimate the behavior of pressure, void fraction, and burst temperature in sprinkler bulbs filled with liquid and its vapor. As before, the glass bulb is assumed to be perfectly rigid and surface tension is neglected. It is further assumed that phase change contributes negligibly to the liquid mass, and that the vapor is in equilibrium with the liquid and follows Clausius-Clapeyron behavior. When a void exists, the pressure in the glass bulb is below atmospheric and is expressed as 


$$
\frac{p}{p_{0}}=\exp \left[\frac{h_{f g} M W}{R_{u}}\left(\frac{1}{T_{0}}-\frac{1}{T}\right)\right] .
$$

While a void exists, the void fraction relationship of Eq. 5, developed for an air void, is valid. This means that Fig. 2 also applies for a glass bulb filled with liquid and its vapor.

As temperature increases, the void shrinks until it disappears. The lowest temperature for which there is no void, defined as $T_{d i s}$, comes from Eq. 5 and is:

$T_{\text {dis }}=T_{0}+\beta^{-1}\left[\alpha_{0} /\left(1-\alpha_{0}\right)+\left(p_{\text {dis }}-p_{0}\right) / K\right]$.

This quantity is plotted in Fig. 4 for a representative glass bulb. Because the burst temperature is expected to be only a few degrees above the void disappearance temperature, Eq. 8 and Fig. 4 represent a reasonable approximation of burst temperature.

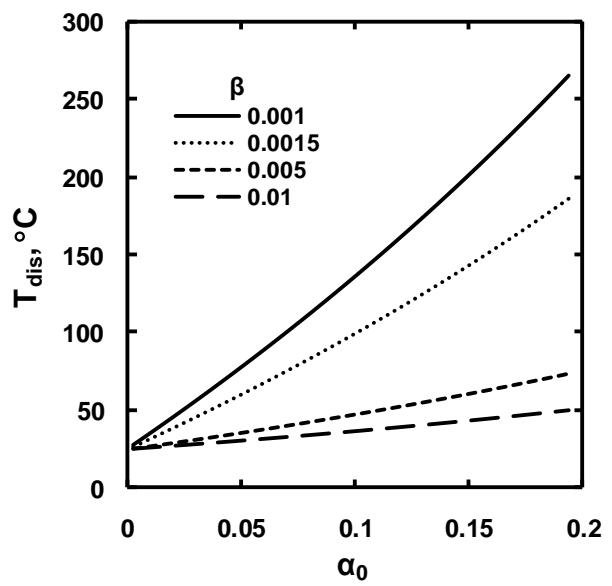

Fig. 4. Bubble disappearance temperature as a function of initial void fraction for a glass bulb filled with liquid and its vapor, where $T_{0}=25^{\circ} \mathrm{C}$ and $K$ is infinite.

If temperature increases above $T_{d i s}$, thermal expansion of the liquid must be balanced by a sharp increase in pressure and/or an increase in the bulb volume. Assuming the glass to be perfectly rigid, this balance can be expressed as:

$\beta\left(T-T_{d i s}\right)=K^{-1}\left(p-p_{d i s}\right)$.

The pressure in a glass bulb is given by Eqs. 6 and 9 for temperatures above and below the void disappearance temperature, respectively. This behavior is plotted in Fig. 5, taking isopropanol as a representative liquid. Comparing Fig. 5 with Fig. 2 reveals that the increase of pressure with temperature is much steeper when the void contains only the vapor phase of the bulb liquid. This results in burst temperatures that are more tightly controlled.

\section{EXPERIMENTAL}

The activation of three different sprinkler types was tested experimentally. The sprinkler models are listed in Table 2 along with the rated $T_{\text {burst }}$ and crush loads from the manufacturers. The composition of the liquids used in these bulbs are not shared by the manufacturers. Sprinkler images are shown in Fig. 6. Dimensions of the sprinkler bulbs were measured at room temperature with a caliper and are summarized in Table 2. In the bulb volume calculation of Viking sprinklers, the interior top and bottom of the bulbs were assumed to be conical, while the center sections were assumed to be cylindrical. For ASCOA sprinklers, the top section was assumed to be spherical, the bottom section was assumed to be conical, and the center section was assumed to be cylindrical. 


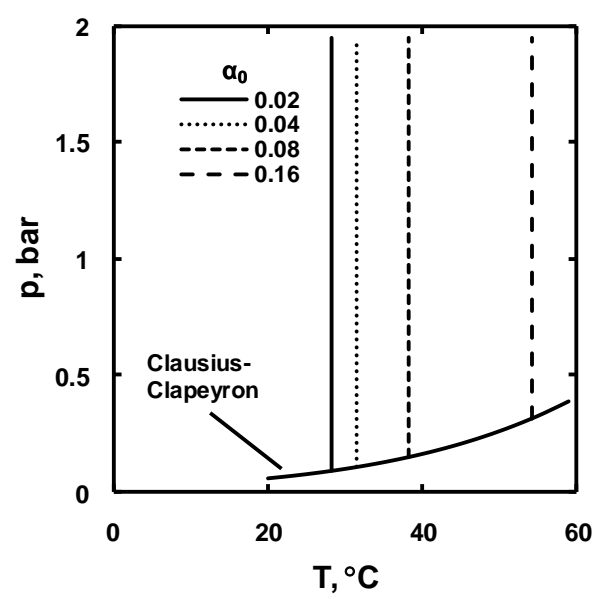

Fig. 5. Pressure as a function of temperature for a bulb filled with isopropanol liquid and vapor.
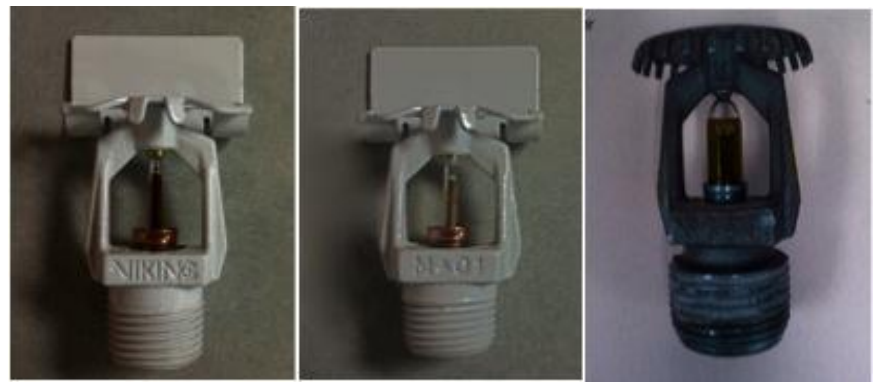

Fig. 6. From left to right: Viking red, Viking yellow, and ASCOA yellow sprinklers (on-line version in color).

Table 2. Manufacturers' specifications and measured bulb dimensions.

\begin{tabular}{cccccc}
\hline Make/model & Rated $\boldsymbol{T}_{\text {burst }} \boldsymbol{~}^{\mathbf{0}} \mathbf{C}$ & Crush load, kN & OD, $\mathbf{c m}$ & Wall, $\mathbf{c m}$ & $\boldsymbol{v}_{\text {bulb }}, \mathbf{c m}^{\mathbf{3}}$ \\
\hline Viking sidewall red & 68 & 3.5 & 0.279 & 0.071 & 0.0197 \\
Viking sidewall yellow & 79 & 3.5 & 0.279 & 0.071 & 0.0197 \\
ASCOA upright yellow & 79 & unknown & 0.793 & 0.097 & 0.498 \\
\hline
\end{tabular}

To measure changes in the size of the gas voids with temperature, the sprinklers were placed at the center of a water bath, as sketched in Fig. 7. The container was filled with water and heated on a hotplate at a rate of approximately $2{ }^{\circ} \mathrm{C} / \mathrm{min}$. Two K-type thermocouples monitored the temperature of the water bath and ensured uniformity of the bath temperature.

The bulbs were imaged with a Nikon D100 single-lens reflex color digital camera with a $60 \mathrm{~mm}$ lens at f/3.3. The lens was positioned $7.5 \mathrm{~cm}$ from the wall of the glass container. An image of the sprinkler bulb and void was taken every $5{ }^{\circ} \mathrm{C}$, until the water temperature was within $10^{\circ} \mathrm{C}$ of the rated burst temperature, at which point an image was taken every $1{ }^{\circ} \mathrm{C}$. A sample image is shown in Fig. 8.

The images were analyzed using paint.net imaging software. The dimensions of the voids were measured as shown in Fig. 8. A measurement of void half width, $a$, was measured along the longest chord, while the void half height, $b$, was measured along the longest perpendicular chord. The gas voids were assumed to be ellipsoidal in shape, with volumes of

$v_{\text {gas }}=4 \pi a^{2} b / 3$. 
The gas volumes were converted to void fractions using Eq. 1.

Uncertainties in the measured bulb temperatures are estimated at $\pm 1.5^{\circ} \mathrm{C}$. Uncertainties in the void fractions, which arise primarily from effects of light refraction and the assumed ellipsoidal void shape, are estimated at $\pm 20 \%$. Effects of hysteresis were examined and were found to be negligible.

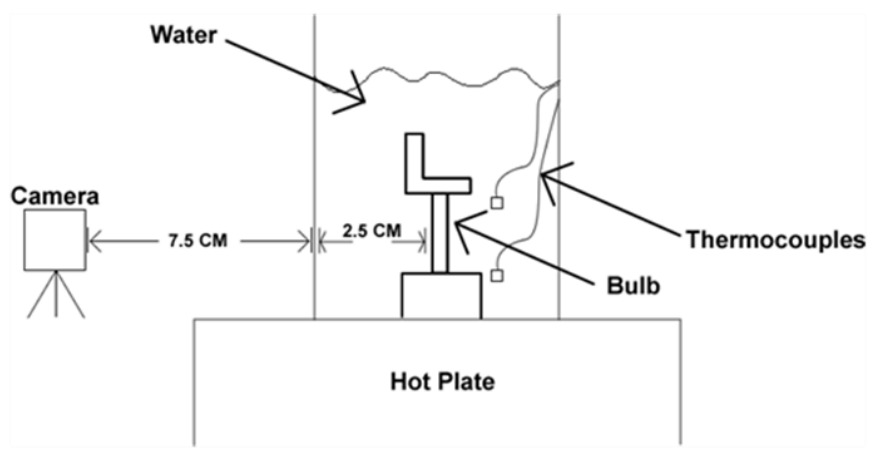

Fig. 7. Experimental schematic.

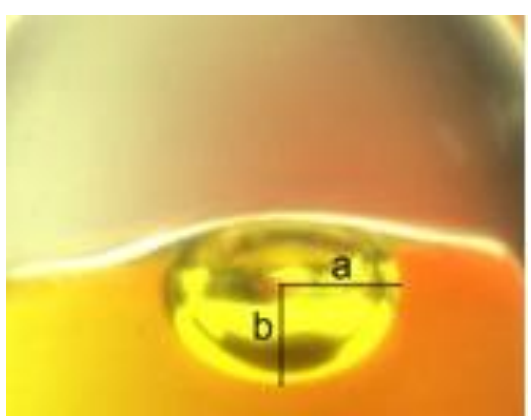

Fig. 8. Image of bubble with dimensions (on-line version in color).

\section{RESULTS}

Measured void fractions are plotted with respect to temperature for the Viking bulbs in Figs. 9a and 9b. Two trials are shown for each type. Typical of all the tests considered, the data of Fig. 9 are repeatable. The void fractions of Fig. 9 decrease linearly until void fraction becomes zero. The complete disappearance of the voids indicates that the bulbs of Fig. 9 contain no gas other than vapor from the liquid species.

The slopes of the low temperature regions in Figs. 9a and 9b are denoted $c_{1}$ and shown in Table 3 . Inflections are evident in Fig. 9 where the void disappears. Because there is no gas other than vapor in these bulbs, the pressure is low until the void disappears and Eq. 5 can be applied assuming infinite $K$. Equation 5 thus yields a thermal expansion coefficient of $\beta=0.0020 \mathrm{~K}^{-1}$ for the bulbs of Fig. 9, which is within the range of the liquids considered in Table 1.

The glass bulbs considered in Fig. 9 have voids that disappear prior to burst, with this occurring at a temperature of $T_{\text {dis }}=T_{\text {inflection }}$. This temperature is on average $13{ }^{\circ} \mathrm{C}$ below the measured burst temperature, $T_{\text {burst }}$, as shown in Table 3. Referring to Fig. 4, this indicates that the pressure inside the bulbs increases only slightly until about $13{ }^{\circ} \mathrm{C}$ below burst. If the glass is perfectly rigid until burst, the maximum pressure in the bulb can be found from Eq. 9. Inserting $\beta=0.0020 \mathrm{~K}^{-1}$ and $K=2000 \mathrm{MPa}$ into Eq. 9 indicates a burst pressure of 520 bar. Such a high pressure is improbable given the bulb dimensions of Table 2, thus it is expected that thermal expansion of the glass occurs prior to burst.

Measured void fractions are plotted with respect to temperature for ASCOA bulbs in Fig. 10. The void fractions decrease linearly until a temperature of $T_{\text {inflection }}$. Because the voids do not completely disappear before burst, it can be concluded that these bulbs contain a gas such as air that does not enter the liquid 
Table 3. Summary of behavior in $\alpha$ versus $T$ plots.

\begin{tabular}{ccccccc}
\hline Sprinkler & $\boldsymbol{T}_{\text {inflection }},{ }^{\circ} \mathbf{C}$ & $\boldsymbol{T}_{\text {burst }},{ }^{\circ} \mathbf{C}$ & $\boldsymbol{k}_{\mathbf{1}}$ & $\boldsymbol{k}_{\mathbf{2}}$ & $\boldsymbol{c}_{\mathbf{1}},{ }^{\circ} \mathbf{C}^{-\mathbf{1}}$ & $\boldsymbol{c}_{\mathbf{2}},{ }^{\circ} \mathbf{C}^{-\mathbf{1}}$ \\
\hline Viking red & 55 & 68.5 & 0.105 & 0 & -0.0019 & 0 \\
Viking yellow & 67 & 79 & 0.121 & 0 & -0.0018 & 0 \\
ASCOA yellow & 70 & 79.5 & 0.0187 & 0.00209 & -0.00026 & -0.000026 \\
\hline
\end{tabular}

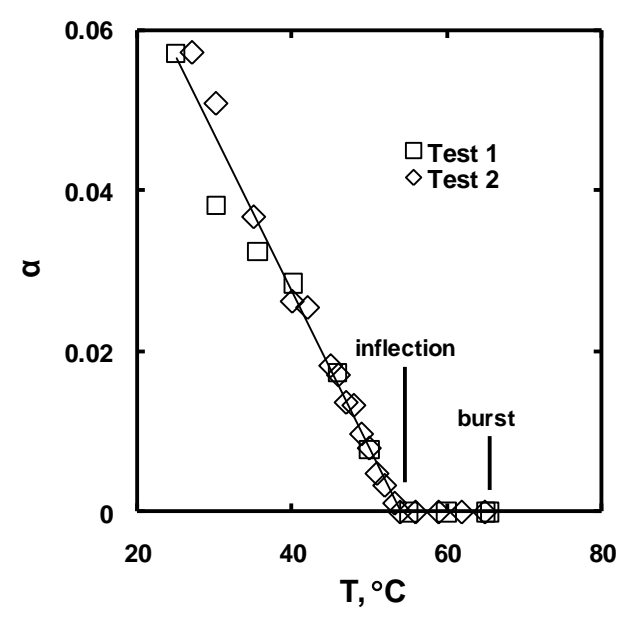

(a)

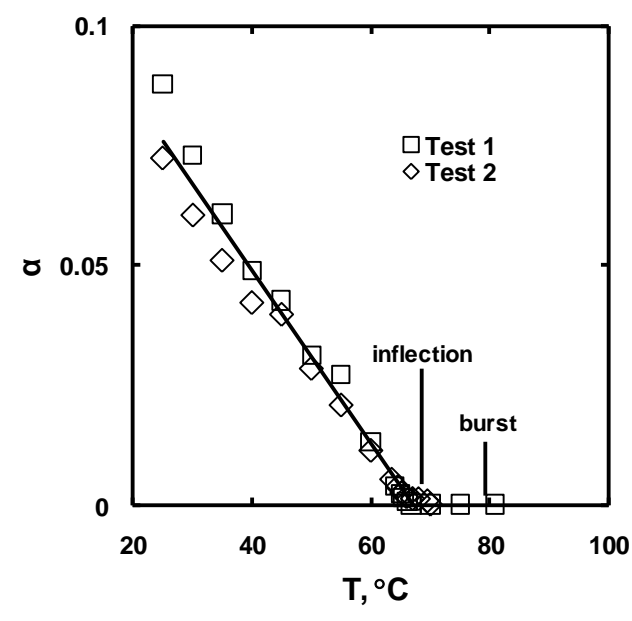

(b)

Fig. 9. Void fraction as a function of temperature for: (a) Viking red bulbs; (b) Viking yellow bulbs.

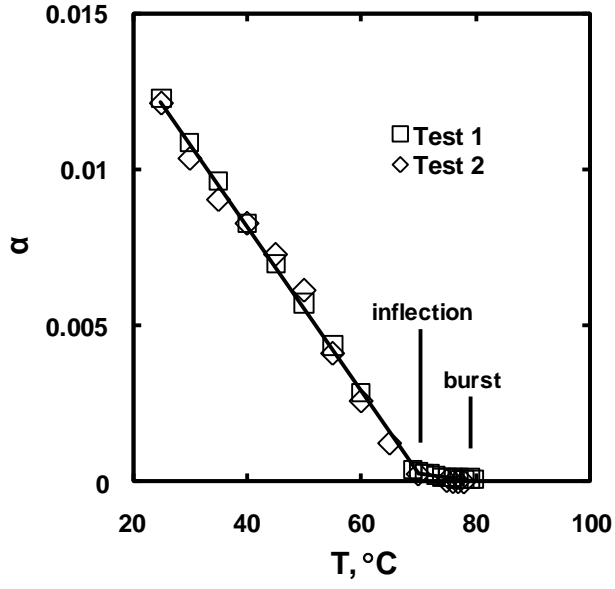

(a)

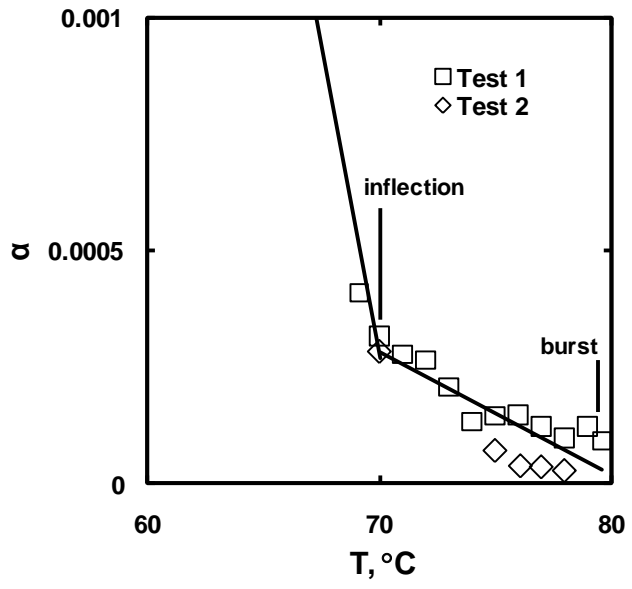

(b)

Fig. 10. Void fraction as a function of temperature for ASCOA Yellow bulbs showing (a) overall behavior and (b) expanded scale at high temperatures.

phase. The presence of an inflection for these bulbs is not explained by the present theoretical analysis and warrants further study.

The trends of Fig. 10 can be divided into two linear regions with different slopes. To capture these trends, the data were fit with piecewise functions of the form: 
$\alpha=\left\{\begin{array}{l}k_{1}+c_{1} T \text { for } T \leq T_{\text {inflection }} \\ k_{2}+c_{2} T \text { for } T>T_{\text {inflection }}\end{array}\right.$.

The fitted function parameters are listed in Table 3 together with the experimentally determined burst temperatures $\left(T_{\text {burst }}\right)$. A comparison of data in Tables 2 and 3 reveals the experimental burst temperatures to be within $1{ }^{\circ} \mathrm{C}$ of the manufacturers' ratings.

\section{CONCLUSIONS}

This study characterizes the behavior of expanding liquids in sprinkler bulbs using both analysis and experiments. The major findings are as follows.

1. The analysis predicts void fractions in sprinkler bulbs to decrease linearly with increasing temperature until the void disappears (for bulbs filled with liquid and its vapor) or the bulb fractures (for bulbs filled with liquid and air). These predictions are generally supported by observations. The slope of this relationship is proportional to the thermal expansion coefficient of the liquid.

2. The analysis predicts the pressure to increase with temperature. However, this increase is much steeper after the void disappears, i.e., for bulbs filled with liquid and its vapor and sufficiently heated.

3. For all glass bulbs, the burst temperature increases with decreasing initial void fraction.

4. For the Viking sprinklers the voids disappeared at about $13{ }^{\circ} \mathrm{C}$ below burst, indicating they are filled with liquid and its vapor. For the ASCOA sprinklers the voids did not disappear prior to burst, indicating they contain liquid and non-condensable gas such as air.

\section{ACKNOWLEDGMENTS}

Helpful discussions with Jose Torero, Ken Isman, and Paul Pinigis, and Mehran Mohammed are greatly appreciated. The two undergraduate authors acknowledge support from the Dept. of Fire Protection Engineering.

\section{REFERENCES}

[1] Fire Protection Handbook, National Fire Protection Association, Quincy, MA, p. 10-172 (2003).

[2] Hague, D.R., Glass Bulb Technology, in Plumbing Systems and Design, p. 28, March, 2004.

[3] Klein, W., "Thermal Triggering Device for Sprinklers for Stationary Fire-Extinguishing Systems," U.S. Patent 4981179, Jan. 1, 1991.

[4] Khan, M.M. and Chaffee, J.L., (2006) Determination of Activation Temperature of Glass Bulb Sprinklers using a Thermal Liquid Bath, Fire and Materials 30: 271-283. http://dx.doi.org/10.1002/fam.909

[5] Ingason, H. (1998) Investigation of Thermal Response of Glass Bulb Sprinklers using Plunge and Ramp Tests, Fire Safety Journal. 30: 71-93. http://dx.doi.org/10.1016/S0379-7112(97)00026-X

[6] Lai, C., Su, H.-C., Tsai, M.-J., Chen, C.J., Tzeng, C.-T., and Lin, T.-H., (2010) Influence of Fire Source Locations on the Actuation of Wet-Type Sprinklers, Building and Environment 45: 107114. http://dx.doi.org/10.1016/j.buildenv.2009.05.007

[7] Rodriguez, H., Williams, M., Wilkes, J.S., and Rogers, R.D., (2008) Ionic Liquids for Liquid-inGlass Thermometers, Green Chemistry, 10: 501-507. http://dx.doi.org/10.1039/B800366A

[8] Dreisbach, R.R. and Shrader, S.A., (1949) Vapor Pressure-Temperature Data on Some Organic Compounds, Ind. Eng. Chem., 41: 2879-2880.

[9] Majer, V. and Svoboda, V., Enthalpies of Vaporization of Organic Compounds: A Critical Review and Data Compilation, Blackwell Scientific Publications, Oxford, 1985, 300. 
[10] NFPA 13: Standard for Installation of Sprinkler Systems, National Fire Protection Association, Quincy, MA, 2010. 